\title{
Dynamic Resting-State Functional Connectivity in Major Depression
}

\author{
Roselinde H Kaiser ${ }^{*,}$, Susan Whitfield-Gabrieli', Daniel G Dillon', Franziska Goer', Miranda Beltzer', \\ Jared Minkel ${ }^{3,4}$, Moria Smoski ${ }^{3}$, Gabriel Dichter ${ }^{4}$ and Diego A Pizzagalli*, \\ 'Department of Psychiatry, Harvard Medical School, and Center for Depression, Anxiety and Stress Research, McLean Hospital, Belmont, MA, \\ USA; ${ }^{2}$ Department of Brain and Cognitive Sciences, Massachusetts Institute of Technology, Cambridge, MA, USA; ${ }^{3}$ Department of Psychiatry and \\ Behavioral Sciences, Duke University Medical Center, Durham, NC, USA; ${ }^{4}$ Department of Psychiatry, University of North Carolina at Chapel Hill \\ School of Medicine, Chapel Hill, NC, USA
}

\begin{abstract}
Major depressive disorder (MDD) is characterized by abnormal resting-state functional connectivity (RSFC), especially in medial prefrontal cortical (MPFC) regions of the default network. However, prior research in MDD has not examined dynamic changes in functional connectivity as networks form, interact, and dissolve over time. We compared unmedicated individuals with MDD $(n=100)$ to control participants $(n=109)$ on dynamic RSFC (operationalized as SD in RSFC over a series of sliding windows) of an MPFC seed region during a resting-state functional magnetic resonance imaging scan. Among participants with MDD, we also investigated the relationship between symptom severity and RSFC. Secondary analyses probed the association between dynamic RSFC and rumination. Results showed that individuals with MDD were characterized by decreased dynamic (less variable) RSFC between MPFC and regions of parahippocampal gyrus within the default network, a pattern related to sustained positive connectivity between these regions across sliding windows. In contrast, the MDD group exhibited increased dynamic (more variable) RSFC between MPFC and regions of insula, and higher severity of depression was related to increased dynamic RSFC between MPFC and dorsolateral prefrontal cortex. These patterns of highly variable RSFC were related to greater frequency of strong positive and negative correlations in activity across sliding windows. Secondary analyses indicated that increased dynamic RSFC between MPFC and insula was related to higher levels of recent rumination. These findings provide initial evidence that depression, and ruminative thinking in depression, are related to abnormal patterns of fluctuating communication among brain systems involved in regulating attention and self-referential thinking.

Neuropsychopharmacology (2016) 4I, I822-1830; doi:I0.I038/npp.20I5.352; published online 23 December 20I5
\end{abstract}

\section{INTRODUCTION}

Major depressive disorder (MDD) is a debilitating psychiatric illness that affects one in six people (Kessler et al, 2012), and is a leading cause of mortality worldwide (Ferrari et al, 2013). People suffering from MDD exhibit abnormal communication among brain systems (Kaiser et al, 2015), especially in midline and temporal areas of the default network that are recruited together for self-referential and internally-directed attention (Andrews-Hanna et al, 2014). Theoretical models of depression point to such aberrations as key to the cognitive and emotional dysfunctions of MDD (Marchetti et al, 2012; Whitfield-Gabrieli and Ford, 2012), and propose that disrupted brain communication is central to the pathophysiology of the disorder.

*Correspondence: Dr RH Kaiser or DA Pizzagalli, Center for Depression, Anxiety and Stress Research, McLean Hospital, I I 5 Mill St, Belmont, MA 02478, USA, Tel: +6178554234 or +617855423I, Fax:+6178554230, E-mail: RHKaiser@mclean.harvard.edu or DAP@mclean.harvard.edu

Received I3 October 2015; revised 20 November 20I5; accepted 30 November 2015; accepted article preview online 3 December 2015
One method for examining brain communication is through the analysis of resting-state functional connectivity (RSFC), measured as correlations in the activity of discrete brain regions during rest (Biswal et al, 1995). A number of studies have examined static RSFC, or dimensions of brain communication that are relatively stable over time, by extracting overall functional connectivity across an extended period of rest. However, an emerging area of study is dynamic RSFC (Hutchison et al, 2013a), or dimensions of brain communication that change over time, which can be investigated by measuring variability in the strength or spatial organization of functional connectivity among brain regions. Much remains unknown about the neurocognitive significance of dynamic RSFC, but early work suggests that changes in functional connectivity can be related to changes in vigilance (Thompson et al, 2013), arousal (Chang et al, 2013a), or emotional state (Cribben et al, 2012), and emerge across species and states of consciousness (Hutchison et al, 2013b). Critically, the magnitude of RSFC variability appears to be a reliable property that depends in part on functional relationships between neural systems (Hutchison et al, 2013a). Brain regions with common functions (eg, bilateral 
homologues or brain regions within prototypical (static) functional networks) tend to show lower dynamic RSFC with one another (Meunier et al, 2009; Shen et al, 2015; Supplementary Figure S1). Regions that are broadly involved in higher-order regulatory functions (eg, prefrontal cortical areas) show higher dynamic RSFC, a pattern that may relate to flexible coupling of regulatory systems with other brain networks either at rest or in the service of goal-directed processing (Bray et al, 2015; Gonzalez-Castillo et al, 2014). Thus, an updated view of RSFC integrates both static and dynamic components: the static component represents stable dimensions of overall RSFC, and the dynamic component represents the processes by which networks and subnetworks coalesce and dissolve over time, or crosstalk between networks.

The investigation of dynamic RSFC may provide new insights into abnormal brain communication in MDD. Previous research conducted with individuals with schizophrenia (Ma et al, 2014), Alzheimer's disorder (Jones et al, 2012), or bipolar disorder (Rashid et al, 2014), revealed altered dynamic RSFC in these patient populations. For example, compared to healthy controls, patients with schizophrenia exhibited decreased variability over time in the spatial configuration of the default network, but increased variability in the coordinated activity of frontoparietal and temporal regions ( $\mathrm{Ma}$ et al, 2014). Patients with Alzheimer's disorder showed more persistent activity and functional connectivity among anterior regions of the default network, eg, medial prefrontal cortex (MPFC), than age-matched controls (Jones et al, 2012). Although dynamic RSFC is an emerging area in psychiatry, these studies suggest that altered dynamic RSFC, particularly in areas of the default network, may be an important dimension of the pathophysiology of psychiatric illnesses.

The nature of dynamic RSFC that characterizes MDD may be complex, and depend on functional relationships between brain systems. A recent meta-analysis of static RSFC in depression revealed increased RSFC among regions of the default network, especially in MPFC (Kaiser et al, 2015). Amplified static RSFC among default network regions may be accompanied by decreased dynamic RSFC, perhaps together reflecting the habit of inflexible, sustained attention to self-referential thoughts that is central to depressive rumination. The meta-analysis also showed that depressed individuals exhibited abnormal static RSFC between regions of the default network and areas of prefrontal cortex or insula believed to regulate attention on the basis of abstract goals or salient events ((Kaiser et al, 2015; Menon and Uddin, 2010; Nelson et al, 2010; Zanto and Gazzaley, 2013), but see Craig (2009) or Gasquoine (2014) for other perspectives on insular functioning, and see Chang et al (2013b) for discussion of functional subregions of insula). Empirical findings converge with this meta-analytic result, showing that depression was related to larger increases in functional connectivity between areas of the default network and prefrontal systems in response to emotionally negative distractors (Kaiser et al, 2014). Collectively, this meta-analytic and empirical work suggests that the pathophysiology of MDD may include increased dynamic RSFC between midline (default network) and prefrontal or insular (regulatory) systems. Such increased dynamic RSFC could be related to depressive rumination as well, eg, may reflect a tendency for intrusive, self-focused thoughts to distract attention from goals. In sum, evidence for abnormal dynamic RSFC may be consistent with evidence for depression-related changes in static RSFC, but would provide a nuanced view of temporal qualities of brain network communication that complements previous work.

To examine dynamic RSFC in depression, we tested two hypotheses in a sample of unmedicated adults with MDD and non-psychiatric control participants. First, we predicted that in comparison to control participants, individuals with MDD would exhibit decreased RSFC variability between MPFC, a hub of the default network, and other regions of the default network. Second, we predicted that individuals with MDD would show amplified RSFC variability between MPFC and regulatory systems, eg, areas of insula putatively involved in allocating resources toward or away from the default network (Sridharan et al, 2008). To examine depression from a dimensional perspective, we also investigated dynamic MPFC connectivity as a function of depressive symptom severity within the MDD group. Secondary analyses conducted in a subsample of participants (who completed measures of recent rumination) tested the hypothesis that higher levels of rumination would be associated with altered dynamic RSFC in regions implicated by depression.

\section{MATERIALS AND METHODS}

\section{Participants}

Participants were unmedicated depressed adults (MDD group, $n=100$, age $19-63, M=31.30$ years, and $61.00 \%$ female) who met DSM-IV-TR criteria for current MDD and healthy controls (control group, $n=109$, age 18-64, $\mathrm{M}=31.54$ years, and $62.39 \%$ female). Participants were recruited at three research sites (McLean Hospital, Harvard University, and Duke University/University of North Carolina at Chapel Hill) and gave written informed consent approved by each site's Institutional Review Board. For all analyses, dummy-coded covariates for each site were included to control for potential confounding effects of scanner, scanning protocol, or sample characteristics (Supplementary Tables S1 and S2). Portions of data collected at Duke/UNC have been reported in a recent publication testing static resting-state predictors of treatment response (Crowther et al, 2015); data from the McLean and Harvard sites have not been published before. Psychiatric history was assessed with the Structured Clinical Interview for DSM-IV-TR Axis I Disorders. Exclusion criteria for the MDD group included symptoms of psychosis or mania; other comorbid axis I disorders were permitted if secondary to the MDD diagnosis (see Supplementary Table S1 for demographics and clinical characteristics). Exclusion criteria for the control group included any major lifetime axis I disorders. Controlling for current or previous psychiatric diagnosis co-occurring with MDD (eg, anxiety) failed to affect statistical findings. For both groups, participants reporting a history of head injury, neurological disorders, major medial illnesses, or current pregnancy were excluded. 


\section{Procedure}

Imaging procedure. See Supplementary Table S2 for details on data acquisition. Participants completed an eyes-open resting functional MRI scan of $5.0 \mathrm{~min}$ (Duke/UNC) to $6.2 \mathrm{~min}$ (McLean, Harvard). A high-resolution T1-weighted anatomical image was acquired prior to functional scanning for the purpose of normalization and coregistration of functional data.

Depressive symptoms. Severity of depression was assessed using the Beck Depression Inventory, Second Edition (BDI-II; Beck et al, 1996). Scores were mean-deviated by subtracting the average BDI-II score from each individual score.

Rumination. At the Duke/UNC site only, rumination was assessed using the Behavioral Activation for DepressionAvoidance Subscale (BADS-AV; Kanter et al, 2007). This self-report scale provides a measure of recent (past two weeks) rumination. Scores were mean-deviated by subtracting the average BADS-AV score from each individual score.

\section{Analysis of fMRI Data}

General image preprocessing. The first $6 \mathrm{~s}$ of each participant's functional data were discarded to allow for stabilization of the magnetic field. Functional data were preprocessed in SPM8 (http://www.fil.ion.ucl.ac.uk/spm/soft ware/spm8/), using the standard spatial preprocessing steps of slice-time correction, realignment, normalization in Montreal Neurological Institute (MNI) space, and smoothing with a 6-mm kernel.

Head motion and artifact detection. Head motion was assessed by translation and rotation in $x, y$, and $z$ directions using SPM8. For each participant, time points of significant head motion or fluctuations in the magnetic field $(>1 \mathrm{~mm}$ motion from previous frame, global mean intensity $>3 \mathrm{SD}$ from mean intensity across functional scans) were calculated using Artifact Detection Tools (ART, www.nitrc.org/pro jects/artifact_detect/). There were no differences between groups or sites in number of outlier volumes (Supplementary Table S2). Outlier images were modeled in the first-level general linear model (as one vector per outlier time point, each containing 1 for the corresponding outlier and $0 \mathrm{~s}$ for all other time points) to remove the influence of these time points on estimates of functional connectivity while maintaining the temporal structure of the data. Also modeled in the first-level model were residual head motion parameters (three translation and three rotation parameters, plus one composite motion parameter reflecting the maximum scanto-scan movement) and their first-order derivatives. There were no differences between groups or sites in estimates of composite motion or framewise displacement.

Functional connectivity analysis. Voxelwise seed-based functional connectivity analyses were performed using the CONN toolbox (https://www.nitrc.org/projects/conn/; Whitfield-Gabrieli and Nieto-Castanon, 2012) and in-house scripts written in Matlab (Mathworks, Natick, MA). The seed region of interest (ROI) was based on a bilateral medial prefrontal region that exhibited depression-related abnormalities in RSFC in a recent meta-analysis of data (non-overlapping with the present study) from 556 individuals with MDD and 518 healthy controls (Kaiser et al, 2015). The seed ROI was a mask created from results of the meta-analysis, gray-matter-masked to restrict seeds to cortex, and falls within the default network (Yeo et al, 2011).

Physiological and other sources of noise were estimated and regressed out using CompCor (Behzadi et al, 2007), a method that performs principal component analysis to estimate the physiological noise from white matter and cerebrospinal fluid for each participant. Next, a temporal band-pass filter of $0.0278-0.10 \mathrm{~Hz}$ was applied to the time series. This range was selected to remove high-frequency activity related to cardiac and respiratory activity (Cordes et al, 2001), and low-frequency activity with a period that exceeds the duration of sliding windows used in dynamic analyses (Leonardi and Van De Ville, 2015). In sum, detrending, outlier censoring, motion regression, and CompCor correction were performed simultaneously in a single first-level regression model, followed by band-pass filtering. These corrections yielded a residual BOLD time course at each voxel that was used for subsequent analyses.

Dynamic analyses. For first-level dynamic analyses, the time course was segmented into $36-\mathrm{s}$ windows (see recommendation by Leonardi and Van De Ville, 2015), sliding the onset of each window by $18 \mathrm{~s}$, for a total of 15-19 windows (depending on length of the functional scan). The duration of sliding windows was selected to optimize the balance between capturing rapidly shifting dynamic relationships (with shorter windows) and achieving reliable estimates of the correlated activity between regions (with longer windows; Leonardi and Van De Ville, 2015). Next, the Fisher's $z$-transformed Pearson's correlation coefficient was computed for each sliding window between the truncated time course of the seed and that of all other voxels, yielding a set of sliding-window beta maps for each participant. Dynamic connectivity was estimated by calculating the SD in beta values at each voxel.

Group-level dynamic analyses were conducted by entering first-level dynamic maps into a whole-brain regression analysis and performing group-level statistics on the SD in beta values at each voxel. To test the hypotheses that the MDD group and the control group differed on dynamic RSFC of MPFC, a $t$-test was performed at each voxel. Correlation analyses to examine the relationship between severity of depression and dynamic RSFC were conducted within the MDD group by performing a correlation between mean-deviated BDI-II scores and dynamic connectivity at each voxel. Group-level effects were considered significant if they exceeded a peak amplitude of $p<0.01$ (two-sided), cluster corrected within a gray matter brain mask to false discovery rate of $p<0.05$ (see Supplementary Table S3 for a summary of dynamic analytic methods). Analyses were also repeated including age, sex, co-occurring psychiatric diagnosis, and number of outlier images as group-level covariates; because controlling for these variables did not affect results, simple analyses (with site covariates, only) are reported. 


\section{Secondary Analyses}

Descriptive statistics for window-by-window RSFC. To clarify the underlying patterns of RSFC across sliding windows that contributed to significant group-level effects, we computed descriptive statistics to examine the frequency of positive or negative correlations between the seed ROI and the region of effect. Specifically, for each participant, we calculated the proportion of windows in which (Fisher's $z$-transformed Pearson) correlations between the seed ROI and the region of effect fell within particular ranges: high negative $(z<-0.5)$, moderate negative $(-0.5 \leqslant z<-0.25)$, low/uncorrelated $(-0.25 \leqslant z \leqslant 0.25)$, moderate positive $(0.25<z \leqslant 0.5)$, and high positive $(z>0.5)$. Next, we computed the average proportion of windows in which RSFC fell within each range for the MDD group and the control group, and correlated BDI-II scores with the proportion of windows in each range of RSFC.

Analyses to examine recent rumination and dynamic $R S F C$. We conducted analyses to explore the association between recent rumination and dynamic RSFC in regions implicated by depression. To accomplish this, we extracted dynamic RSFC values from each cluster of significant effect from primary analyses and performed multiple regressions to test direct and interactive effects of BADS-AV on dynamic RSFC. Because depression factors (group, or BDI-II) were used to define clusters of abnormal dynamic RSFC, it was important to include appropriate depression factors in each regression to avoid circularity ( $\mathrm{Vul}$ et al, 2009). Therefore, rumination analyses were performed as follows. In regression analyses secondary to whole-brain group contrasts, group (contrast coded as MDD $=+1$, control $=-1$ ), rumination (mean-deviated BADS-AV), and the interaction of these variables were regressed on dynamic RSFC extracted from clusters implicated by group $t$-tests. If a significant interaction between rumination and group was detected, simple regressions were performed within the MDD group and the control group using BADS-AV to predict dynamic RSFC. In regression analyses secondary to whole-brain correlation analyses, depression severity (BDI-II), rumination (BADS-AV), and the interaction of these variables were regressed on dynamic RSFC extracted from clusters implicated by correlation analyses. Outlier checks were performed by calculating Cook's D (Cook, 1977) and excluding observations that had undue influence according to the standard threshold (Cook's $D>4 / n$; Bollen and Jackman, 1985); this approach resulted in no more than three observations excluded from any regression.

Static analyses. To determine whether dynamic RSFC and static RSFC provide overlapping or complementary information, we also performed whole-brain static analyses. For firstlevel static analyses, the Fisher's $z$-transformed Pearson's correlation coefficient was computed between the full time course of the MPFC seed and the time course of all other voxels. Group-level static RSFC analyses were performed by entering first-level static maps into a whole-brain regression analysis and performing group-level $t$-tests (between the MDD group and the control group) or correlations (with BDI-II scores) at each voxel.

\section{RESULTS}

Our findings indicate that, consistent with predictions, depression was related to decreased dynamic (less variable) RSFC between MPFC and other regions of the prototypical default network, but increased dynamic (more variable) RSFC between MPFC and regions of insula and lateral prefrontal cortex.

Compared with the control group, the MDD group exhibited decreased dynamic RSFC between MPFC and regions of parahippocampal gyrus (PHG; Figure 1a and b; Table 1). Secondary analyses showed that invariable connectivity between MPFC and PHG was related to decreased frequency of windows in which activity was uncorrelated, ie, for depressed participants, positive connectivity rarely dissolved between these regions (Figure 1c; Supplementary Figure S2A). Relative to the control group, the MDD group also showed higher dynamic RSFC between MPFC and a region of right insula (Figure 1d and e; Table 1). This pattern of hypervariability in MDD was related to more frequent windows of highly correlated activity (positive or negative correlations) between MPFC and insula (Figure 1f; Supplementary Figure S2B).

Next, we performed analyses to test the association between severity of depression and dynamic RSFC within the MDD group. Results showed that higher severity of depression was associated with more variable connectivity between MPFC and dorsolateral prefrontal cortex (DLPFC) (Figure 2a and b; Table 1), a pattern related to greater frequency of sliding windows in which activity in these regions was highly correlated (positive or negative correlations; Figure 2c; Supplementary Figure S2C).

No differences were observed between research sites in these analyses (Supplementary Figure S3).

We performed secondary regression analyses to explore the relationship between rumination and dynamic RSFC in the regions implicated by primary analyses, ie, PHG (Figure 3a), insula (Figure 3b), and DLPFC (Figure 3c). Results revealed a significant interaction between recent rumination and group in predicting dynamic RSFC between MPFC and insula, $\mathrm{F}(1,38)=4.76, p=0.03$. Specifically, higher levels of recent rumination were associated with increased dynamic RSFC for individuals with $\mathrm{MDD}, \mathrm{F}(1,21)=7.91$, $p=0.01$, but not among control participants, $\mathrm{F}(1,17)=1.56$, $p=0.23$. Regression analyses did not yield significant effects of recent rumination on dynamic RSFC between MPFC and either the PHG or DLPFC, $p s>0.1$.

Finally, we performed whole-brain static RSFC analyses using the same MPFC seed. These analyses failed to reveal significant effects at thresholds selected for primary analyses, but at a lower peak threshold $(p<0.05$, two-tailed, FDR $p<0.05$, a threshold which meets the premise of random field theory but detects larger clusters of lower-magnitude effects (Friston et al, 1996)); higher depression severity was associated with higher static RSFC between MPFC and areas of dorsal posterior cingulate (Supplementary Figure S4; Supplementary Table S4). No group differences in static RSFC between MDD and control participants were detected at either peak threshold. 


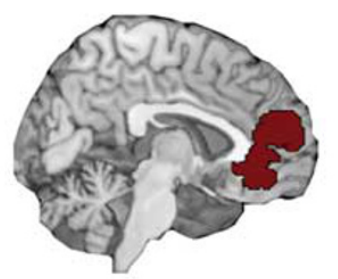

Seed ROI

in MPFC

\section{Dynamic Functional Connectivity in PHG}

a

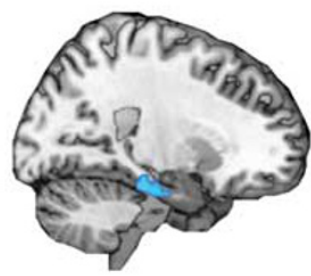

b

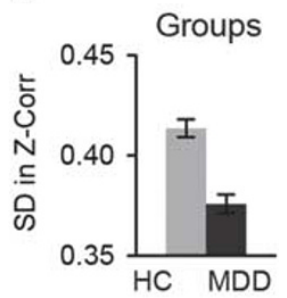

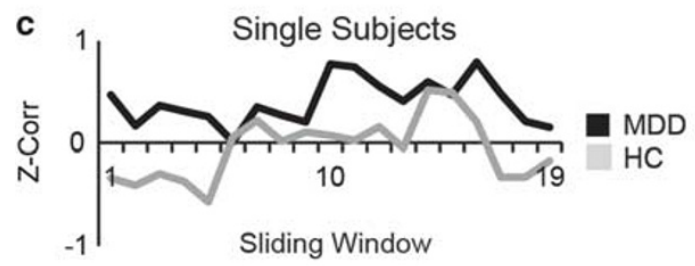

Dynamic Functional Connectivity in Insula d

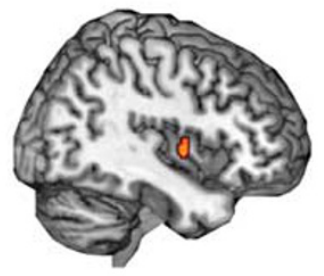

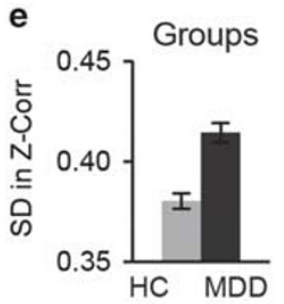

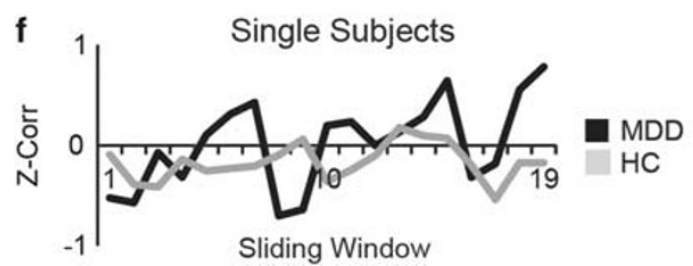

Figure I Dynamic resting-state functional connectivity of medial prefrontal cortex (MPFC) in unmedicated individuals with major depressive disorder (MDD) compared with healthy controls (HC). (a) Compared to the HC group $(n=109)$, the MDD group $(n=100)$ exhibited decreased dynamic functional connectivity between a seed region of interest (ROI) in MPFC and regions of parahippocampal gyrus (PHG). (b) Average dynamic functional connectivity (SD in Fisher's $z$-transformed Pearson's correlations across a series of sliding windows) in the PHG for each group. (c) To illustrate window-by-window patterns of functional connectivity, displayed are MPFC-PHG functional connectivity values (Fisher's z-transformed Pearson's correlations) for each of the 19 sliding windows, for a single subject in the MDD group and a single subject in the HC group. (d) In comparison to the HC group, the MDD group also had increased dynamic functional connectivity between MPFC and reas of insula. (e) Average dynamic functional connectivity in the insula for each group. ( $f$ ) To illustrate window-by-window patterns of functional connectivity, shown are MPFC-insula functional connectivity values for each of the 19 sliding windows, for a single subject in the MDD group and a single subject in the $\mathrm{HC}$ group. Note: group differences (MDD - HC) are significant at peak amplitude $p<0.01$, two-tailed $t$-test, FDR $p<0.05$.

\section{DISCUSSION}

This study provides evidence of abnormal dynamic RSFC in MDD and at higher levels of self-reported depression severity. Previous studies have linked MDD to disrupted static RSFC, especially among regions of the default network, frontal regions involved in regulating activity in other neural systems (Kaiser et al, 2015). The present results complement this prior work by showing that MDD is characterized by abnormal patterns of fluctuating functional connectivity in overlapping regions. Here, depression was associated with persistent RSFC among medial prefrontal and temporal regions of the default network (Yeo et al, 2011), but more variable and amplified RSFC between MPFC and anterior insula or DLPFC.

One interpretation of the present findings is that abnormal dynamic RSFC underlies the negative, dysregulated thinking style that characterizes depression. The anterior insula is a region believed to use salience information to guide allocation of resources to other networks (Menon et al, 2010; Sridharan et al, 2008). Of note, experimentally induced sadness has been related to increases in the coordinated activity of insula and medial frontal regions (Mayberg et al, 1999). Therefore, highly variable MPFC-to-insula connectivity in depressed individuals may signify increased sensitivity to salient emotional or self-referential information that triggers rumination. In support of this idea, higher levels of recent rumination were associated with increased variability in functional connectivity between MPFC and insula for depressed individuals. Invariable functional connectivity between MPFC and PHG may also reflect self-focused thinking, as synchronized activity in these systems is believed to support autobiographical memory or prospection (Andrews-Hanna et al, 2010). Although persistent RSFC between MPFC and PHG was not related to recent rumination, it may be related to a trait tendency to dwell on past negative events. Finally, highly variable MPFC-to-DLPFC connectivity may signify weaknesses in brain circuits responsible for cognitive control (Banich et al, 2000; MacDonald et al, 2000), and could be related to depressive deficits in executive functioning such as difficulty inhibiting emotional distraction. These interpretations, however, are speculative, and evidence presented here for a relationship between rumination and dynamic RSFC should be viewed as preliminary because this relationship could only be tested in a subsample of individuals $(n=45)$ at a single site.

An important question is whether dynamic RSFC provides unique information about communication among neural systems beyond that provided by static RSFC. Greater magnitude of static RSFC typically implies lower variability in RSFC (Chang and Glover, 2010), hence these dimensions of functional connectivity may usually have an inverse relationship. However, group differences in overall correlated activity may occur despite similar levels of variability, just as distinct profiles of variability may be present in different groups but overall correlations appear the same. Here, unique regions of the prototypical default network were implicated by depression in dynamic $v s$ static analyses, and depression-related changes in dynamic (but not static) RSFC were observed between MPFC and brain regions that fall outside the default network. Together, this suggests that 
Table I Dynamic Resting-State Functional Connectivity of Medial Prefrontal Cortex

\begin{tabular}{|c|c|c|c|c|c|c|c|}
\hline \multirow[b]{3}{*}{ MDD compared with HC } & \multirow{3}{*}{$\begin{array}{c}\text { Peak coord } \\
\\
x, y, z\end{array}$} & \multirow{3}{*}{$\begin{array}{c}\text { Peak } t \\
t\end{array}$} & \multirow{3}{*}{$\begin{array}{l}\text { Vol } \\
\# \mathbf{v x}\end{array}$} & \multicolumn{4}{|c|}{ Dynamic FC of cluster } \\
\hline & & & & \multicolumn{2}{|c|}{ MDD } & \multicolumn{2}{|c|}{$\mathrm{HC}$} \\
\hline & & & & M & SD & $\mathbf{M}$ & SD \\
\hline Insula & $32,-4,-2$ & 3.91 & 101 & 0.42 & 0.06 & 0.37 & 0.05 \\
\hline Correlation with BDI-II & & & & $r$ & & & \\
\hline DLPFC & $50,28,10$ & 4.31 & 132 & 0.21 & & & \\
\hline
\end{tabular}

Abbreviations: BDI-II, Beck Depression Inventory, Second Edition; coord, coordinates in MNI standard stereotaxic space; DLPFC, dorsolateral prefrontal cortex; FC, functional connectivity; $\mathrm{HC}$, healthy control; MDD, major depressive disorder; vol, volume of cluster of significant effect in voxels; $v \times$, number of $|\times| \times \mid$ mm voxels. Thresholding was set to $p<0.01$ (two-tailed) peak amplitude of effect, cluster corrected to a false discovery rate of $p<0.05$. Dynamic FC was assessed by SD in Fisher's $z$-transformed Pearson's correlations across sliding windows.

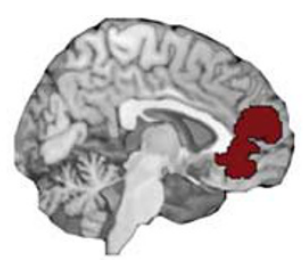

Seed ROI

in MPFC

\section{Dynamic Functional Connectivity in DLPFC}

a

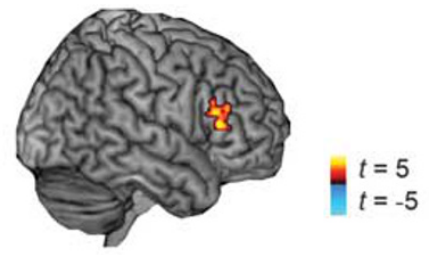

c

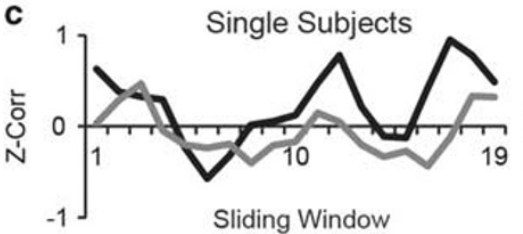

b 0.55 MDD Group

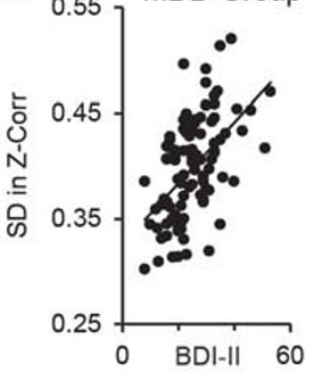

High BDI-II Low BDI-II

Figure 2 Dynamic resting-state functional connectivity of medial prefrontal cortex (MPFC) as a function of depressive symptom severity. (a) For depressed individuals ( $n=97$; three participants did not fill out the Beck Depression Inventory, Second Ed (BDI-II)), higher scores on the BDI-II were associated with amplified dynamic functional connectivity between a seed region of interest (ROI) in MPFC and areas of dorsolateral prefrontal cortex (DLPFC). (b) Correlation between BDI-II and dynamic functional connectivity (SD in Fisher's z-transformed Pearson's correlations across a series of sliding windows). (c) To illustrate window-by-window patterns of functional connectivity, displayed are MPFC-DLPFC functional connectivity values (Fisher's z-transformed Pearson's correlation) for each of the 19 sliding windows, for a single major depressive disorder (MDD) subject reporting high severity depression $(\mathrm{BDI}-\|=53)$ and a single MDD subject reporting low severity depression $(\mathrm{BDI}-\mid-1=8)$. Note: correlations with $\mathrm{BDI}-\|$ are significant at peak amplitude $p<0.01$, two-tailed t-test, FDR $p<0.05$.

static and dynamic methods can provide complementary information about brain communication in depression.

The present study has some limitations. First, as noted previously, much is unknown about what dynamic RSFC represents in terms of neurocognitive functioning. For example, it remains unknown to what extent abnormalities in RSFC are intrinsic properties, or are influenced by in-the-moment cognitive activities (for discussion of the putative physiological underpinnings of RSFC and clinical implications see Buckner et al (2013); Hutchison et al (2013b); Kaiser et al (2014)). As the field of dynamic RSFC grows, we may gain a better understanding of how these properties of brain functioning relate to cognitive activities and psychopathology. More generally, in light of the novelty of this analytic approach, it will be important to replicate the present findings in an independent sample.

Second, the present study focused on identifying brain regions that showed abnormal variability in RSFC in MDD, but it may be useful to combine this analytic approach with other neurobiological methods to gain a more complete view of the pathophysiology of MDD. For example, other techniques for examining dynamic RSFC (Allen et al, 2014; Rashid et al, 2014) may be applied in conjunction with the present approach to explore variability in both magnitude and spatial extent of RSFC. Alternately, comparing structural and functional measures may provide insight into the relationships between functional connectivity and volumetric abnormalities in depression (van Tol et al, 2014). Studies that collect data in several modalities may illuminate the complex nature of brain communication in depression.

Third, although we adopted both categorical (presence $v s$ absence of MDD) and dimensional (severity of depression) perspectives on depression, additional qualities of depressive illness may be important. MDD is a heterogeneous disorder, and unique patterns of abnormal dynamic connectivity may 

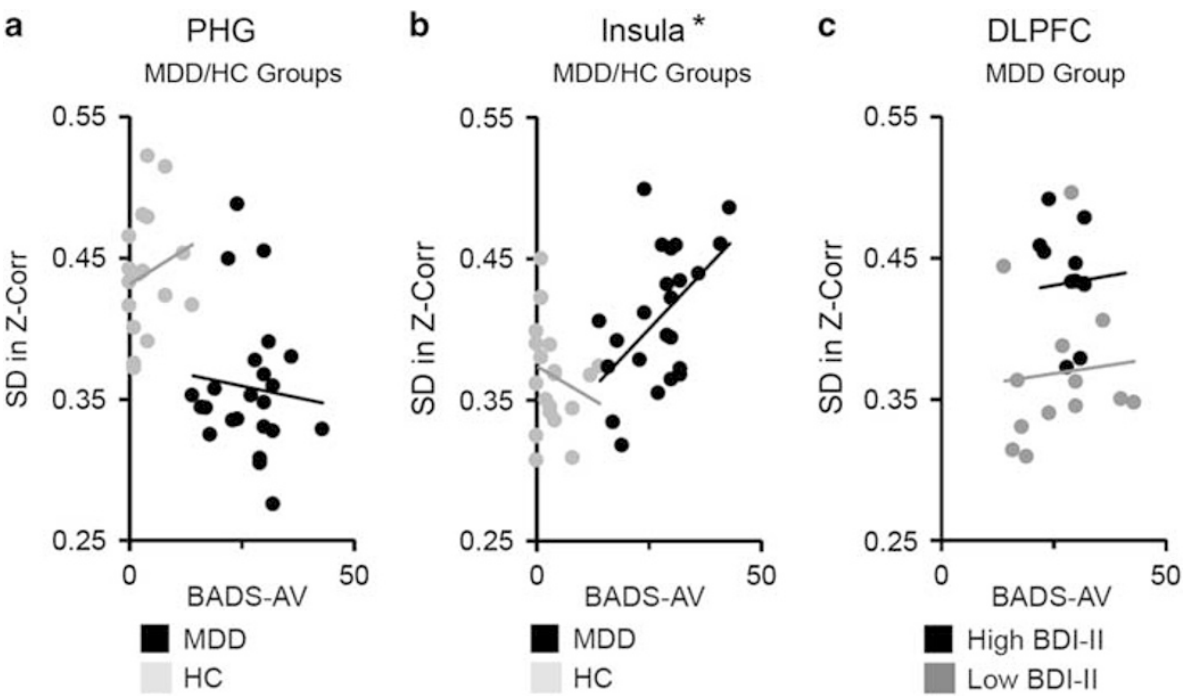

Figure 3 Rumination and dynamic resting-state functional connectivity of medial prefrontal cortex (MPFC). Multiple regression was used to investigate the relationship between rumination and dynamic resting-state functional connectivity between MPFC and regions implicated by the group contrast of participants with major depressive disorder (MDD) vs healthy control (HC) participants (parahippocampal gyrus (PHG) or insula), or regions implicated by depressive symptom severity as measured by the Beck Depression Inventory, Second Ed (BDI-Il; dorsolateral prefrontal cortex (DLPFC)). Analyses were performed in a subset of the sample $(n=45)$ who completed a self-report measure of recent rumination, the Behavioral Activation for Depression-Avoidance subscale (BADS-AV) at the Duke/UNC site. (a) Group (MDD coded as +1 ; HC coded as - I), recent rumination (mean-deviated BADS-AV), and the interaction between these factors were regressed on dynamic resting-state functional connectivity values (SD in Fisher's z-transformed Pearson's correlations across a series of sliding windows) extracted from PHG. No significant effects of rumination were detected. (b) Group, BADS-AV, and their interaction were regressed on dynamic functional connectivity values extracted from insula. Results showed a significant interaction between group and rumination, and follow-up simple regression analyses revealed that in the MDD group (but not the $\mathrm{HC}$ group), higher levels of recent rumination were associated with higher variability in MPFC-to-insula functional connectivity $(* p<0.05)$. (c) Within the MDD group, BDI-II, BADS-AV, and their interaction, were regressed on dynamic functional connectivity values extracted from DLPFC. No significant effects of rumination were detected. Note: in panel c, the MDD group is median-split on BDI-II scores for display purposes, only. Analyses were performed using continuous measures.

emerge for different subtypes of MDD or stages of illness. Furthermore, although the BDI-II and BADS-AV are wellvalidated measures of depression severity and recent rumination, respectively (Beck et al, 1996; Kanter et al, 2007), they represent only one (self-report) facet of depression and cognitive style. Future studies may capture additional dimensions of illness such as clinician-rated depression severity or behavioral measures of emotion regulation.

Fourth, this cross-sectional study cannot address causal associations between RSFC and MDD. For example, it could be that erratic coordination among medial and lateral prefrontal systems is a risk factor for deficits regulating attention or mood, which in turn make an individual vulnerable in developing depression. Alternately, it may be that as negative thoughts repeatedly co-opt attention systems, a pattern of more variable coupling between midline and lateral prefrontal regions is established in the brain. Future longitudinal studies may begin to parse such causal relationships.

In conclusion, this resting-state study provides evidence that the presence and severity of MDD is associated with abnormal dynamic functional connectivity. Increased variability among prefrontal and insular regions may be related to biased salience processing or interruptions to cognitive control, whereas decreased variability among areas of the default network may be associated with the persistence of internally directed attention. These findings offer a new perspective on RSFC in MDD, and suggest directions for future research on fluctuating dynamic neural communication in mood disorders.

\section{FUNDING AND DISCLOSURE}

Over the past 3 years, DAP reports having received honoraria/consulting fees from Otsuka America Pharmaceutical and Pfizer for activities unrelated to this project. RHK, SW-G, DGD, JM, MS, and GD, and FG and MB, report no biomedical financial interests.

\section{ACKNOWLEDGMENTS}

We thank Alfonso Nieto-Castanon for valuable discussion of analytic approach, and Min Su Kang for help in manuscript preparation. This work was partially supported by the Rappaport Mental Health Research Fellowship and National Institute of Mental Health (NIMH) grant F32 MH106262 awarded to RHK, NIMH grant R00 MH094438 awarded to DGD, NIMH grants R21 MH094781 and R21 MH094781 S1 awarded to MS and GD, and NIMH grants R01 MH068376 and R01 MH101521 awarded to DAP.

\section{REFERENCES}

Allen EA, Damaraju E, Plis SM, Erhardt EB, Eichele T, Calhoun VD (2014). Tracking whole-brain connectivity dynamics in the resting state. Cereb Cortex 24: 663-676.

Andrews-Hanna JR, Reidler JS, Sepulcre J, Poulin R, Buckner RL (2010). Functional-anatomic fractionation of the brain's default network. Neuron 65: 550-562.

Andrews-Hanna JR, Smallwood J, Spreng RN (2014). The default network and self-generated thought: component processes, 
dynamic control, and clinical relevance. Ann NY Acad Sci 1316: 29-52.

Banich MT, Milham MP, Atchley RA, Cohen NJ, Webb A, Wszalek $\mathrm{T}$ et al (2000). Prefrontal regions play a predominant role in imposing an attentional 'set': evidence from fMIRI. Cogn Brain Res 10: 1-9.

Beck AT, Steer RA, Ball R, Ranieri WF (1996). Comparison of Beck Depression Inventories-IA and -II in psychiatric outpatients. J Pers Assess 67: 588-597.

Behzadi Y, Restom K, Liau J, Liu TT (2007). A component based noise correction method (CompCor) for BOLD and perfusion based fMRI. Neuroimage 37: 90-101.

Biswal B, Yetkin FZ, Haughton VM, Hyde JS (1995). Functional connectivity in the motor cortex of resting human brain using echo-planar MRI. Magn Reson Med 34: 537-541.

Bollen KA, Jackman RW (1985). Regression diagnostics: an expository treatment of outliers and influential cases. Sociol Methods Res 13: 510-542.

Bray S, Arnold A, Levy RM, Iaria G (2015). Spatial and temporal functional connectivity changes between resting and attentive states. Hum Brain Mapp 36: 549-565.

Buckner RL, Krienen FM, Yeo BTT (2013). Opportunities and limitations of intrinsic functional connectivity MRI. Nat Neurosci 16: $832-837$

Chang C, Glover GH (2010). Time-frequency dynamics of restingstate brain connectivity measured with fMRI. Neuroimage 50: 81-98.

Chang C, Metzger CD, Glover GH, Duyn JH, Heinze HJ, Walter M (2013a). Association between heart rate variability and fluctuations in resting-state functional connectivity. Neuroimage 68: 93-104.

Chang LJ, Yarkoni T, Khaw MW, Sanfey AG (2013b). Decoding the role of the insula in human cognition: functional parcellation and large-scale reverse inference. Cereb Cortex 23: 739-749.

Cook RD (1977). Detection of influential observation in linear regression. Technometrics 19: 15-18.

Cordes D, Haughton VM, Arfanakis K, Carew JD, Turski PA, Moritz CH et al (2001). Frequencies contributing to functional connectivity in the cerebral cortex in "resting-state" data. AJNR Am J Neuroradiol 22: 1326-1333.

Craig AD (2009). How do you feel-now? The anterior insula and human awareness. Nat Rev Neurosci 10: 59-70.

Cribben I, Haraldsdottir R, Atlas LY, Wager TD, Lindquist MA (2012). Dynamic connectivity regression: determining state-related changes in brain connectivity. Neuroimage 61: 907-920.

Crowther A, Smoski MJ, Minkel J, Moore T, Gibbs D, Petty C et al (2015). Resting-state connectivity predictors of response to psychotherapy in major depressive disorder. Neuropsychopharmacology 40: 1659-1673.

Ferrari AJ, Charlson FJ, Norman RE, Patten SB, Freedman G, Murray CJL et al (2013). Burden of depressive disorders by country, sex, age, and year: findings from the Global Burden of Disease Study 2010. PLoS Med 10: 12.

Friston KJ, Holmes A, Poline JB, Price CJ, Frith CD (1996). Detecting activations in PET and fMRI: levels of inference and power. Neuroimage 4: 223-235.

Gasquoine PG (2014). Contributions of the insula to cognition and emotion. Neuropsychol Rev 24: 77-87.

Gonzalez-Castillo J, Handwerker DA, Robinson ME, Hoy CW, Buchanan LC, Saad ZS et al (2014). The spatial structure of resting state connectivity stability on the scale of minutes. Front Neurosci 8: 19.

Hutchison RM, Womelsdorf T, Allen EA, Bandettini PA, Calhoun VD, Corbetta M et al (2013a). Dynamic functional connectivity: promise, issues, and interpretations. Neuroimage 80: $360-378$
Hutchison RM, Womelsdorf T, Gati JS, Everling S, Menon RS (2013b). Resting-state networks show dynamic functional connectivity in awake humans and anesthetized macaques. Hum Brain Mapp 34: 2154-2177.

Jones DT, Vemuri P, Murphy MC, Gunter JL, Senjem ML, Machulda MM et al (2012). Non-stationarity in the "resting brain's" modular architecture. PLoS One 7: 15.

Kaiser RH, Andrews-Hanna JR, Spielberg JM, Warren SL, Sutton BP, Miller GA et al (2014). Distracted and down: neural mechanisms of affective interference in subclinical depression. Soc Cogn Affect Neurosci 10: 654-663.

Kaiser RH, Andrews-Hanna JR, Wager TD, Pizzagalli DA (2015). Large-scale network dysfunction in major depressive disorder: a meta-analysis of resting-state functional connectivity. JAMA Psychiatry 72: 603-611.

Kanter JW, Mulick PS, Busch AM, Berlin KS, Martell CR (2007). The Behavioral Activation for Depression Scale (BADS): psychometric properties and factor structure. J Psychopathol Behav Assess 29: 191-202.

Kessler RC, Petukhova M, Sampson NA, Zaslavsky AM, Wittchen HU (2012). Twelve-month and lifetime prevalence and lifetime morbid risk of anxiety and mood disorders in the United States. Int J Methods Psychiatr Res 21: 169-184.

Leonardi N, Van De Ville D (2015). On spurious and real fluctuations of dynamic functional connectivity during rest. Neuroimage 104: 430-436.

Ma S, Calhoun VD, Phlypo R, Adali T (2014). Dynamic changes of spatial functional network connectivity in individuals and schizophrenia patients using independent vector analysis. Neuroimage 90: 196-206.

MacDonald AW, Cohen JD, Stenger VA, Carter CS (2000). Dissociating the role of the dorsolateral prefrontal and anterior cingulate cortex in cognitive control. Science 288: $1835-1838$.

Marchetti I, Koster EHW, Sonuga-Barke EJS, De Raedt R (2012). The default mode network and recurrent depression: a neurobiological model of cognitive risk factors. Neuropsychol Rev 22: 229-251.

Mayberg HS, Liotti M, Brannan SK, McGinnis S, Mahurin RK, Jerabek PA et al (1999). Reciprocal limbic-cortical function and negative mood: converging PET findings in depression and normal sadness. Am $J$ Psychiatry 156: 675-682.

Menon V, Uddin LQ (2010). Saliency, switching, attention and control: a network model of insula function. Brain Struct Funct 214: 655-667.

Meunier D, Lambiotte R, Fornito A, Ersche K, Bullmore E (2009). Hierarchical modularity in human brain functional networks. Front Hum Neurosci 3: 37.

Nelson SM, Dosenbach NUF, Cohen AL, Wheeler ME, Schlaggar BL, Petersen SE (2010). Role of the anterior insula in task-level control and focal attention. Brain Struct Funct 214: 669-680.

Rashid B, Damaraju E, Pearlson GD, Calhoun VD (2014). Dynamic connectivity states estimated from resting fMRI identify differences among schizophrenia, bipolar disorder, and healthy control subjects. Front Hum Neurosci 8: 13.

Shen K, Hutchison RM, Bezgin G, Everling S, McIntosh AR (2015). Network structure shapes spontaneous functional connectivity dynamics. J Neurosci 35: 5579-5588.

Sridharan D, Levitin DJ, Menon V (2008). A critical role for the right fronto-insular cortex in switching between central-executive and default-mode networks. Proc Natl Acad Sci USA 105: 12569-12574.

Thompson GJ, Magnuson ME, Merritt MD, Schwarb H, Pan WJ, McKinley A et al (2013). Short-time windows of correlation between large-scale functional brain networks predict vigilance 
intraindividually and interindividually. Hum Brain Mapp 34: 3280-3298.

van Tol MJ, Li M, Metzger CD, Hailla N, Horn DI, Li W et al (2014). Local cortical thinning links to resting-state disconnectivity in major depressive disorder. Psychol Med 44: 2053-2065.

Vul E, Harris C, Winkielman P, Pashler H (2009). Puzzlingly high correlations in fMRI studies of emotion, personality, and social cognition. Perspect Psychol Sci 4: 274-290.

Whitfield-Gabrieli S, Ford JM (2012). Default mode network activity and connectivity in psychopathology. In: NolenHoeksema S (ed).
Annual Review of Clinical Psychology, Vol. 8. Annual Reviews: Palo Alto, pp 49.

Whitfield-Gabrieli S, Nieto-Castanon A (2012). Conn: a functional connectivity toolbox for correlated and anticorrelated brain networks. Brain Connect 2: 125-141.

Yeo BTT, Krienen FM, Sepulcre J, Sabuncu MR, Lashkari D, Hollinshead $\mathrm{M}$ et al (2011). The organization of the human cerebral cortex estimated by intrinsic functional connectivity. J Neurophysiol 106: 1125-1165.

Zanto TP, Gazzaley A (2013). Fronto-parietal network: flexible hub of cognitive control. Trends Cogn Sci 17: 602-603.

Supplementary Information accompanies the paper on the Neuropsychopharmacology website (http://www.nature.com/npp) 